\title{
Energía solar en paradas de bus una aplicación moderna y vanguardista
}

\section{(Solar energy in bus stops a modern and avant-garde application)}

\author{
Héctor Sebastián Naranjo Silva ${ }^{1}$, Diego Javier Punina Guerrero ${ }^{2}$, Juan José Morales Martinez ${ }^{3}$ \\ ${ }^{1}$ Universidad Politécnica de Cataluña, Barcelona, España \\ ${ }^{2}$ Universidad Técnica Estatal de Quevedo, Quevedo, Ecuador \\ ${ }^{3}$ Instituto Tecnológico de Estudios Superiores de Monterrey, Monterrey, México. \\ hector.sebastian.naranjo@upc.edu.com,dpuninag2@uteq.edu.ec,juan_jose370@hotmail.com
}

Resumen: La eficiencia energética es un tema relevante globalmente debido a la creciente dificultad para acceder a fuentes de energía con mínimos impactos ambientales, según la Agencia Internacional de la Energía en el 2016 el porcentaje de renovables mundialmente fue 24,5\% [9], en este sentido el objeto del artículo es transmitir la investigación de un grupo multidisciplinario que se respalda con la ingeniería para determinar condiciones de energía renovable en un proyecto piloto, el proyecto sobre el acondicionamiento de energía fotovoltaica aplicada en paradas de buses eliminando energía por fuentes convencionales a lo largo del boulevard Albino Corzo, en la ciudad de Chiapas en el departamento de Tuxtla Gutiérrez de México.

La metodología del estudio es cuantitativa basada en resultados de cálculo carácter investigativo y de acción, los resultados son aceptables, contrastados con la incorporación de un arreglo de cinco (5) paneles solares simulados en un programa informático para la parada de bus tipo conejo que reduce $48,37[\mathrm{~kg}]$ de $\mathrm{CO} 2$ anuales al ambiente.

Palabras clave: Chiapas, energía, fotovoltaica, México, panel solar.

\begin{abstract}
Energy efficiency is a relevant issue globally due to the increasing difficulty of accessing energy sources with minimal environmental impacts, according to the International Energy Agency in 2016 the percentage of renewables worldwide was 24.5\% [9], In this sense, the object of the article is to transmit the research of a multidisciplinary group that is supported by engineering to determine renewable energy conditions in a pilot project, the project on the conditioning of photovoltaic energy applied at bus stops eliminating energy from conventional sources along the Albino Corzo boulevard, in the city of Chiapas in the Tuxtla Gutierrez department of Mexico.
\end{abstract}

The study methodology is quantitative based on calculation results of an investigative nature and action, the results are acceptable, contrasted with the incorporation of an arrangement of five (5) simulated solar panels in a computer program for the rabbit type bus stop that reduces $48.37[\mathrm{~kg}]$ of $\mathrm{CO} 2$ per year to the environment.

Keywords: Chiapas, energy, photovoltaic, Mexico, solar panel.

\section{INTRODUCCIÓN}

La Organización de las Naciones Unidas crearon los Objetivos de Desarrollo Sostenible (ODS) como un llamado adoptar medidas para mitigar paulatinamente la pobreza, proteger el planeta, garantizar la paz y prosperidad [14], en dichos objetivos el no. 7 enunciado "Garantizar el acceso a una energía asequible, segura, sostenible y moderna para todos" se basa en fomentar 
el acceso universal a servicios de energía modernas, con mejoras en el rendimiento y que aumenten el uso de fuentes renovables [14]. En este sentido, actualmente ahorrar energía por medio de fuentes de energía renovables es primordial, las políticas de los estados Latinoamericanos y mundiales se centran en impulsar el uso y aplicaciones de tecnologías limpias.

Con las ideas anteriores, al ser la energía solar una de las principales fuentes que se convierte en electricidad útil [12], el artículo investigativo verifica la reducción del consumo proveniente de combustibles fósiles de la red de distribución eléctrica por la integración de un arreglo de paneles solares dentro del sistema de distribución como mecanismo renovable.

El gasto por concepto de pago de energía fósil se trata en todos los países de sustituir por energía amigable con el medio ambiente, en México cerca del $90 \%$ del territorio nacional presenta una irradiación solar [18] (magnitud utilizada para describir la potencia incidente por unidad de superficie $\left[\mathrm{W} / \mathrm{m}^{2}\right]$.) que al día fluctúa entre 5 y $6[\mathrm{kWh}]$ por metro cuadrado, lo que representa hasta un $70 \%$ más alto comparado con los grandes desarrollos de aprovechamiento solar a nivel global [18].

La importancia del estudio es promover la ciudad de Chiapas como pionera en México y América Latina en diseñar este sistema, debido a que en ciudades como Londres (Inglaterra) y Nueva York (USA) está en práctica el uso de estaciones solares en parques de recreación para cargar celulares, pero, esta investigación va más allá, busca implementar luz de lámparas led para iluminar el espacio de la parada y los anuncios luminosos utilizando energía solar por medio de un arreglo de paneles instalados en la parte superior de las paradas de bus.

\section{METODOLOGÍA}

\subsection{Enfoque de la metodología}

La investigación es cuantitativa, se orienta a resultados que se obtienen de la integración de varias etapas de estudio del sistema renovable de energía, analizando datos sobre información histórica comparable, tabulada y medible.

\subsection{Tipos de investigación con referencia a la metodología seleccionada}

Mediante el enfoque seleccionado se utilizan dos tipos de metodologías específicas nombradas a continuación:

- Investigación de acción: es el tipo que conecta el enfoque con programas y acciones permiten encontrar las diferencias entre las variables de estudio para comprobar aspectos, posibilidades y mejoras [2]. El objeto para este tema de análisis es reducir costos a la red pública del sistema energético mediante el uso de paneles fotovoltaicos.

- Investigación bibliográfica: se utiliza con relación a la búsqueda de información del problema mediante manuales, libros, textos de apoyo, internet, y demás relacionado al estudio de la producción eléctrica de sistemas solares [7].

\subsection{Detalle de la investigación}

El proyecto se enfoca a implementar un sistema de energía renovable en un espacio público, como son las paradas de buses de tipo conejo de la avenida central de Chiapas en México. La autogeneración de energía por medio de paneles solares proporciona electricidad promoviendo la aplicación de energía limpia, innovación y con un impacto positivo a nivel regional [2].

La energía es central para los grandes desafíos y oportunidades a los que hace frente el mundo actualmente, sea para uso doméstico, industrial, producción alimenticia, educación, seguridad y hasta para mitigación del cambio climático, en tal razón el acceso global a la energía es esencial. 
La energía sostenible es una oportunidad que transforma la vida, la economía y el planeta, el proyecto se enfoca en un diseño compacto y económico para beneficiar al gobierno y ciudadanos aumentando la producción de energía eléctrica de manera renovable [14].

La situación energética expone que la carga de energía en [kWh] por bimestre sin el prototipo calculado requiere de:

- 3 reflectores de $100[\mathrm{~W}]$.

- 4 focos de $40[\mathrm{~W}]$ tipo incandescente.

La carga neta por los reflectores es 300 [W], los 4 focos 160 [W], por lo tanto, la carga total es de 640 [W] con un uso de 12 horas al día, además con un consumo mensual promedio de 30,4 días al año se tiene una energía necesaria de 167,80 [kWh].

\subsection{Procedimiento}

El procedimiento se basa en levantar los datos de consumo de la parada tipo de Chiapas, posterior calcular los equipos necesarios de energía solar fotovoltaica para subministrar la misma cantidad energética y finalmente se compara mediante un programa computacional de simulación que los equipos y cálculos sean similares.

\section{RESULTADOS}

A continuación, se plasma la estrategia efectuada, la cual comprende dos ítems fundamentales:

1. Reemplazo de subministro de energía de la red pública por paneles solares que generan un sistema fotovoltaico amigable al ambiente.

2. Reemplazo de la luminaria interior por iluminación tipo led más eficiente.

El cálculo la energía en $[\mathrm{kWh}]$ que se consume al bimestre con el prototipo, contiene la siguiente carga:

- 2 tiras en rollo led tipo carrete 5.050 de 5 [m] con consumo de 72 [W].

- 3 focos led tipo rgb de consumo de 20 [W].

Se calcula una carga neta por tiras led en 144 [W], las 3 lámparas en 60 [W] y los cargadores considerando un factor de utilización del $70 \%$ se tiene un total de 0,01 [W], entonces la carga total es de $204[\mathrm{~W}]$ con un uso de 12 horas al día.

Considerando que la luminaria solo se activa por las noches mediante un controlador de carga de corriente directa, esto es un consumo mensual de $74,42[\mathrm{kWh}]$ con el consumo de energía actual.

El consumo anual es de $\$ 5.724$ pesos mexicanos aproximadamente \$287 dólares americanos [22], lo que analizado a 25 años de vida útil del proyecto es de $\$ 150.246$ pesos mexicanos dando $\$ 7.527$ dólares americanos [22], es relevante considerar que se tiene un incremento del $5 \%$ anual en el $[\mathrm{kWh} / \$$ costo], [3].

El potencial de ahorro con el uso del proyecto es de $1.121[\mathrm{kWh}]$ anuales, lo que representa una reducción de consumo de energía del 44,35\%, y con los siguientes cálculos beneficiosos.

\subsection{Cálculo de paneles solares del sistema:}

Para el número de paneles solares se calcula la carga media diaria crítica, con la siguiente fórmula [19]: 


$$
L_{m d}=\frac{L_{m d . D C}+\frac{L_{m d . A C}}{n_{i n v}}}{n_{b a t} \times n_{c o n}}
$$

Dónde:

Lmd es el consumo de energía media diaria;

Lmd.DC, es la carga en corriente directa;

Lmd.AC, es la carga en corriente alterna;

ninv, es la eficiencia del inversor;

そbat, es la eficiencia de la batería; y

ncond, es la eficiencia del conductor.

En el proyecto no se tiene cargas en corriente alterna, ni inversores, Lmd.AC y ninv $=0$, Lmd.DC $=2.448[\mathrm{Wh} /$ día $]=74,12 \times 12$, y por defecto la eficiencia nbat será de $95 \%$ y ncond será de $100 \%$, sustituyendo valores el resultado de la formula son:

$$
\begin{gathered}
\text { Lmd }=[(2.448)+(0)] /(0,95 * 1) \\
\text { Lmd }=2.576,84[\mathrm{Wh} / \text { día }]
\end{gathered}
$$

\subsection{Cálculo del número de paneles solares necesarios:}

$$
N_{T}=\frac{L_{m d . c r i t}}{P_{M P P} x H P S_{\text {crit }} x P R}
$$

Donde:

NT, es el número de paneles solares necesarios;

PMPP, es la potencia pico del panel solar $=180,40[\mathrm{~W}]$;

Lmd.crit, es la energía media diaria critica $=2.576,84[\mathrm{Wh} /$ día $]$;

HPS.crit, son las horas de sol pico del mes crítico = 3,34;

$\mathrm{PR}$, es el factor global de funcionamiento $=0,90$.

Se tiene que el mes crítico de irradiación solar según datos de la NASA es diciembre con 3,34 horas pico de sol (HPS) [13], y con el cálculo de la sección a, la carga Lmd crit $=2576,84$ [Wh/día], la potencia pico del panel solar es de 180,4 [W] y según tablas de especificación del modelo Solarworld serie SW-250 tipo poli cristalino de marca Conermex [5], por lo tanto, sustituyendo datos en la ecuación (2) el resultado es:

$$
\begin{gathered}
N T=\frac{2.576,84}{180,4 \times 3,34 \times 0,9} \\
\mathrm{NT}=4,75 \approx 5 \text { paneles solares. }
\end{gathered}
$$




\subsection{Cálculo de ángulo de inclinación de los paneles solares:}

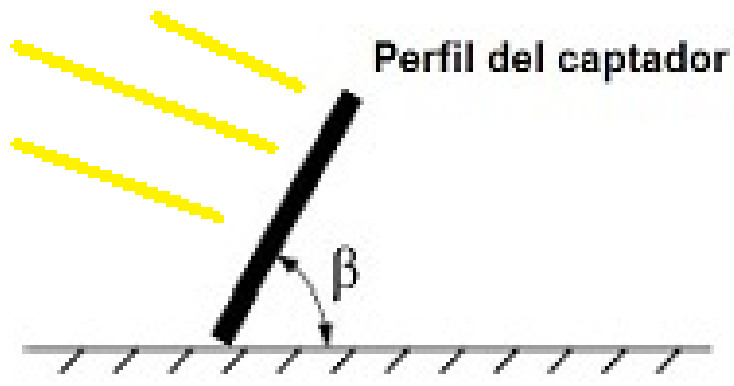

\section{Inclinación del módulo}

Figura 1. Modelo de recepción de luz del panel solar

Fuente: Energía solar y eólica [6].

Se tiene a continuación que la latitud de la ciudad de Chiapas es de $27^{\circ}$, por lo que, según la tabla 1 seguido, se tendrá un ángulo de inclinación para los paneles solares de $27^{\circ}+5^{\circ}=32^{\circ}$ hacia el sur.

Tabla 1. Ángulo de inclinación de paneles solares

Fuente: NASA, Earth Science Enterprise Program [13].

\begin{tabular}{|c|c|}
\hline Latitud del lugar en grados & Ángulo de inclinación \\
\hline $0^{\circ}$ a $15^{\circ}$ & $15^{\circ}$ \\
\hline $15^{\circ}$ a $25^{\circ}$ & La misma latitud \\
\hline $25^{\circ}$ a $30^{\circ}$ & Latitud más $5^{\circ}$ \\
\hline $30^{\circ}$ a $35^{\circ}$ & Latitud más $10^{\circ}$ \\
\hline $35^{\circ}$ a $40^{\circ}$ & Latitud más $15^{\circ}$ \\
\hline $40^{\circ}$ o más & Latitud más $20^{\circ}$ \\
\hline
\end{tabular}

\subsection{Cálculo de banco de baterías:}

Mediante dos parámetros importantes que son la profundidad de descarga máxima y el número de días de autonomía, a continuación, como norma del cálculo del acumulador de energía se toman los parámetros siguientes [11].

- Profundidad de descarga máxima estacional (PDmax,e $)=70 \%=0,7$

- Profundidad de descarga máxima diaria $(\mathrm{PDmax}, \mathrm{d})=15 \%=0,15$

- Número de días de autonomía $(\mathrm{N})=3$

Para evitar insuficiencia se compara la capacidad nominal necesaria de las baterías en función de la profundidad de descarga estacional y diaria, y se selecciona la mayor de ellas. 


\subsection{Capacidad nominal de la batería en función de la descarga máxima diaria (Cnd):}

La explicación de las dos ecuaciones es sencilla, se necesita generar una energía diaria Lmd con las baterías, pero permiten solamente un $15 \%$ de descarga máxima con un factor de corrección de temperatura $(\mathrm{FCT}=1)[8]$.

$$
\begin{gathered}
\boldsymbol{C}_{\boldsymbol{n d}}=\frac{\boldsymbol{L}_{\boldsymbol{m d}}}{\boldsymbol{P}_{\text {Dmax.d }} \boldsymbol{x} \boldsymbol{F}_{\boldsymbol{C T}}} \\
C_{n d}=\frac{2.576,84}{0,15 \times 1}=17.178,93[\mathrm{Wh}]
\end{gathered}
$$

Una vez determina la energía en [Wh] de la batería, simplemente se divide entre la tensión de la misma de 24 [V] en este caso, y se tiene la capacidad mínima para el sistema de acumulación en función de la descarga.

$$
\begin{gathered}
\boldsymbol{C}_{\boldsymbol{n} \boldsymbol{d}}=\frac{\boldsymbol{C}_{\boldsymbol{m d}}[\boldsymbol{W h}]}{\boldsymbol{V}_{\boldsymbol{B A T}}} \\
C_{n d}=\frac{17.178,93}{24}=715,78[\mathrm{Ah}]
\end{gathered}
$$

Además, se calcula la capacidad nominal de la batería en función de la descarga máxima estacional (Cne) [17] en base a:

$$
\begin{gathered}
\boldsymbol{C}_{\boldsymbol{n} \boldsymbol{e}}=\frac{\boldsymbol{L}_{\boldsymbol{m \boldsymbol { d }}} \boldsymbol{x} \boldsymbol{N}}{\boldsymbol{P}_{\boldsymbol{D m a x}, \boldsymbol{e}} \boldsymbol{x} \boldsymbol{F}_{\boldsymbol{C T}}} \\
C_{n e}=\frac{2.576,84 \times 3}{0,7 \times 1}=11.043,60[\mathrm{Wh}] \\
\boldsymbol{C}_{\boldsymbol{n} \boldsymbol{e}}=\frac{\boldsymbol{C}_{\boldsymbol{n} \boldsymbol{e}}[\boldsymbol{W h}]}{\boldsymbol{V}_{\boldsymbol{B A T}}} \\
C_{n e}=\frac{11.043,6}{24}=460[\mathrm{Ah}]
\end{gathered}
$$

\subsection{Cálculo del regulador necesario para el sistema aislado:}

A continuación, el cálculo del regulador, para ello mediante la máxima corriente que soporta el dispositivo a su entrada y salida, además se hace el producto corriente de cortocircuito de un módulo, en este caso del regulador, modelo SW-250 marca Conermex [5] con Isc = 6,96 [A], multiplicado por el número de ramas, donde la corriente de cada rama en paralelo es aproximadamente la misma anteriormente:

$$
\begin{aligned}
& \boldsymbol{C}_{\boldsymbol{n} \boldsymbol{e}}=\mathbf{1 , 2 5} \boldsymbol{x} \boldsymbol{I}_{\boldsymbol{M O D}, \boldsymbol{S C}} \boldsymbol{x} \boldsymbol{N}_{\boldsymbol{p}} \\
& \text { Ientrada }=1,25 \times 6,96 \times 5=43,5 \text { [A] }
\end{aligned}
$$

Donde:

IMOD. SC, es la corriente unitaria del módulo fotovoltaico en condiciones de cortocircuito, en este caso, para el modelo SW-250 es de Isc = 6,96 [A]. 
Complementario se usa la corriente de cortocircuito máxima para el cálculo de entrada al regulador siendo la tensión del módulo fotovoltaico y se cuenta para evitar pérdidas de rendimiento [21].

- $\quad(\mathrm{NP})$ el número de ramas en paralelo = en este caso 5.

- 1,25 es un factor de seguridad para evitar daños ocasionales al regulador.

Para el cálculo de la corriente de salida se valora las potencias de las cargas DC y las cargas AC:

$$
\begin{gathered}
\boldsymbol{C}_{\boldsymbol{n} \boldsymbol{e}}=\boldsymbol{I}_{\text {salida }}=\frac{\mathbf{1 , 2 5} \boldsymbol{x}\left(\boldsymbol{P}_{\boldsymbol{D C}}+\frac{\boldsymbol{P}_{A C}}{\boldsymbol{n i n v}}\right)}{\boldsymbol{V}_{\boldsymbol{B A T}}} \\
I_{\text {salida }}=\frac{1,25(204+0,95)}{24}=10,62[\mathrm{~A}]
\end{gathered}
$$

Dónde: $\mathrm{PDC}=$ potencia de las cargas en corriente continua,

$\mathrm{PAC}=$ potencia de las cargas en corriente alterna; $\mathrm{y}$

ninv $=$ rendimiento del inversor, en torno a $90-95 \%$.

Así pues, el regulador soporta una corriente como mínimo de 44 [A] a su entrada y 11 [A] a su salida.

\subsection{Verificación de cálculo de paneles solares con el sistema informático PVsist 6.3.4:}

Se realiza el diseño y verificación del número de paneles solares en el sistema informático técnico suizo llamado PVsyst V6.3.4, para comparar con el software los cálculos manuales en el literal $b[16]$.

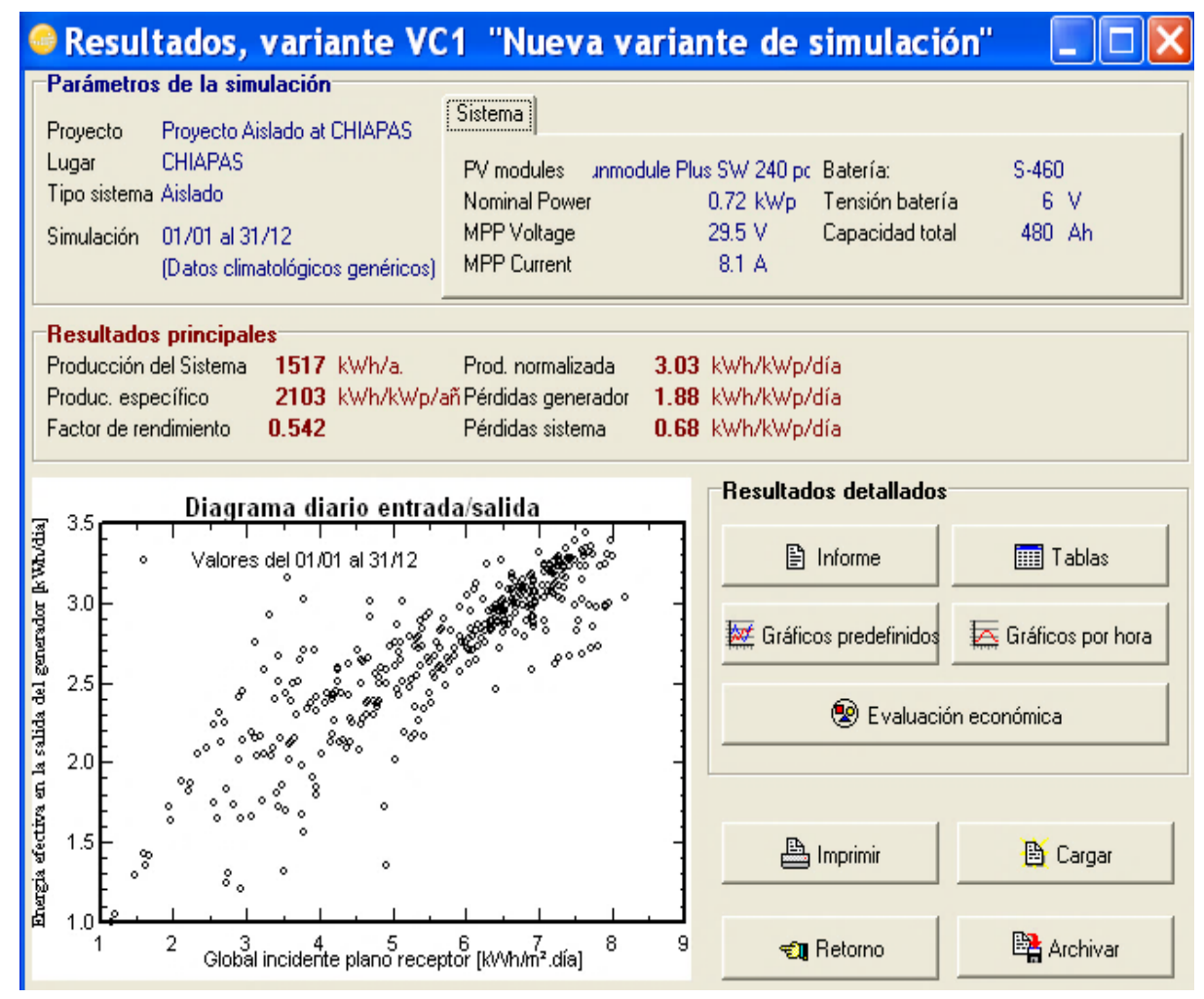

Figura 2. Datos del PVsyst V6.3.4 
Se ingresa la localización, energía necesaria y en base a modelos se determina que existe gran similitud entre ambos, en los cálculos se necesitan 5 paneles solares, mientras que con el software con 4 paneles solares que satisfacen el sistema con la misma capacidad de 480 [Ah] en baterías, y el ángulo de inclinación indica que debe estar a $30^{\circ}$, cálculos afines con la referencia del programa que se ilustra en la figura 2.

\subsection{Valoración económica del proyecto}

A continuación, se analizan y despliegan todos los valores necesarios para la incorporación de la investigación en la tabla 2 siguiente:

Tabla 2. Presupuesto del sistema fotovoltaico

Fuente: Autores

\begin{tabular}{|c|c|c|c|c|c|}
\hline \multirow[b]{2}{*}{ Código } & \multirow[b]{2}{*}{ Concepto } & \multirow[b]{2}{*}{ Unidad } & \multirow[b]{2}{*}{ Cantidad } & \multicolumn{2}{|c|}{ [Pesos mexicanos] } \\
\hline & & & & $\begin{array}{c}\text { Precio } \\
\text { unitario }\end{array}$ & Total \\
\hline $\begin{array}{l}\text { SUM- } \\
\text { COL001 }\end{array}$ & $\begin{array}{l}\text { Suministro y colocación de módulo de } \\
\text { panel solar modelo SW-250 }\end{array}$ & PZA & 5 & 11581,03 & 57905,15 \\
\hline $\begin{array}{l}\text { SUM- } \\
\text { COL002 }\end{array}$ & $\begin{array}{l}\text { Suministro y colocación de batería solar de } \\
24[V] \text {, ciclado profundo marca Surrete } \\
\text { Solar modelo S-480 }\end{array}$ & PZA & 1 & 7327,59 & 7327,59 \\
\hline $\begin{array}{l}\text { SUM- } \\
\text { COL003 }\end{array}$ & $\begin{array}{l}\text { Suministro y colocación de controlador de } \\
\text { carga CIS-10 }\end{array}$ & PZA & 1 & 1379,31 & 1379,31 \\
\hline $\begin{array}{l}\text { SUM- } \\
\text { COL004 }\end{array}$ & $\begin{array}{l}\text { Suministro y colocación de multicargador } \\
\text { de } 10 \text { salidas }\end{array}$ & PZA & 2 & 1200,00 & 2400,00 \\
\hline $\begin{array}{l}\text { SUM- } \\
\text { COL005 }\end{array}$ & $\begin{array}{l}\text { Suministro y colocación de tira led de } 5 \\
\text { [m] de } 72[\mathrm{~W}]\end{array}$ & PZA & 2 & 280,17 & 560,34 \\
\hline $\begin{array}{l}\text { SUM- } \\
\text { COL006 }\end{array}$ & $\begin{array}{l}\text { Suministro y colocación de lámpara led de } \\
20[\mathrm{~W}]\end{array}$ & PZA & 3 & 836,21 & 2508,62 \\
\hline $\begin{array}{l}\text { SUM- } \\
\text { COL007 }\end{array}$ & $\begin{array}{l}\text { Accesorios mixtos necesarios para el } \\
\text { sistema }\end{array}$ & LOTE & 1 & 397,52 & 397,52 \\
\hline $\begin{array}{l}\text { SUM- } \\
\text { COL008 }\end{array}$ & $\begin{array}{l}\text { Suministro y colocación de estructura de } \\
\text { montaje del panel solar (racks) }\end{array}$ & LOTE & 1 & 3103,45 & 3103,45 \\
\hline & & & & $\begin{array}{l}\text { Sub } \\
\text { Total }\end{array}$ & $\$ 75.581,98$ \\
\hline & & & & $\begin{array}{r}16 \% \\
\text { I.V.A. }\end{array}$ & $\$ 12.093,12$ \\
\hline & & & & Total & $\$ 87.675,09$ \\
\hline
\end{tabular}


Homologando al valor en pesos mexicanos con fecha diciembre de 2020 según el Servicio de Administración Tributaria de México para este caso es \$4.392,93 dólares americanos por la implementación del sistema para cada parada de bus [22].

Con el costo de inversión del proyecto, se determina la factibilidad mediante los siguientes métodos [10].

Cálculo de ahorro anual sin el proyecto y con el proyecto.

Horas diarias de consumo $=\frac{50 \% \times 24 \mathrm{hrs}}{100 \%}=\frac{12 \mathrm{hrs}}{\text { día }}$

Si se analiza el consumo anual, donde la carga diaria es de $0,460[\mathrm{~kW}]$, sin el proyecto.

$$
\text { Consumo anual }=\frac{0,46 \mathrm{kw}}{\mathrm{hr}} \times \frac{12 \mathrm{hr}}{1 \mathrm{día}} \times \frac{365 \text { días }}{1 \text { año }}=\frac{2014,80 \mathrm{kwh}}{1 \text { añ } o} \times \frac{2.919 \text { pesos }}{k w h}
$$

Consumo anual $=5881,20$ pesos mexicanos anuales $=322,50$ dólares americanos anuales

Se menciona que, por ser un sistema aislado, los ahorros generados son la facturación al $100 \%$ que se tendría.

\section{DISCUSIÓN}

Cada año en el mundo miles de proyectos de eficiencia energética permanecen sin ser implementados, especialmente en las economías en desarrollo, y fundamentalmente para aquellos países que importan la mayoría de sus recursos energéticos [1].

Una barrera importante para la implementación de dichos proyectos es el financiamiento, debido a que los mecanismos de inversión en eficiencia energética no están completamente desarrollados en las economías locales [2], en este sentido generar estudios y líneas de investigación sobre sistemas energéticos es esencial para apoyar el desarrollo en temas referentes a gestión sostenible energética, mitigación del cambio climático y factores del calentamiento global [12].

Según, el Instituto Nacional de Eficiencia Energética y Energías Renovables de México, con el uso de energía fotovoltaica se tiene que por cada panel solar es equivalente a plantar 9 árboles y sacar de circulación un vehículo durante toda la vida útil del proyecto aproximadamente 25 años [4].

\section{CONCLUSIONES}

Después de los cálculos necesarios se tiene la referencia de reducción de emisiones al ambiente de 48,37 [kg] de CO2 al año mitigados con el reemplazo por el arreglo de paneles solares para paradas de bus.

El proyecto gestiona dos estrategias atractivas para ser sustentable en todos los aspectos técnicos, el primero el remplazo de la fuente de energía de la red pública a modo isla con paneles solares, y el segundo la reconversión tecnológica de luminarias a tecnología led, haciendo que en su base fundamental opere eficientemente.

Se reduce el consumo energía eléctrica de la red pública de 604 [W] a 204 [W] generadas por un arreglo de paneles solares calculados para las condiciones de la ciudad de Chiapas, departamento de Tuxla Gutiérrez en México.

Dicho análisis es un proyecto piloto que se puede aplicar internacionalmente en ciudades donde el potencial de captar energía solar sea alto, abriendo una puerta de mayores análisis y desarrollo energético renovable. 
Es necesario analizar el poder de capación solar en las diversas ciudades y analizar fondos verdes para desarrollar iniciativas de este tipo.

AGRADECIMIENTOS: Se agradece al Municipio de Tuxtla Gutiérrez en México que extendieron datos de la ciudad para coordinar esta investigación, además, se agradece a la Universidad Técnica Estatal de Quevedo por ensanchar las fronteras del conocimiento con este tipo de propuesta académica de difusión que apoyan el desarrollo investigativo.

\section{REFERENCIAS}

[1] Alexandru, M, (2013). Sustainability assessment of electricity generation technologies using weighted multi criteria decision analysis.

[2] Berrío, Luis Humberto; Zuluaga, Carlos, (2014). Smart Grid y la energía solar fotovoltaica para la generación distribuida: una revisión en el contexto energético mundial. Universidad del Norte, Ingeniería y Desarrollo. Vol. 32 n. ${ }^{\circ}$ 2: 369-396, 2014. ISSN: 0122-3461 (impreso). 2145-9371 (on line).

[3] Burns, J, (2009). Solar Energy Processes. New York, EE.UU. John Wiley \& Sons.

[4] Cabrerizo, E, (2012). Instalaciones Solares Fotovoltaicas. Sevilla, España.

[5] Conermex SA, (2015). Energía Solar, soluciones en energías renovables, paneles solares. Disponible en: http://www.conermex.com.mx/

[6] Energía Solar y Eólica, (2015). Energía Fotovoltaica. Disponible en: http://www.eolicaysolar.com/

[7] Gómez-Luna, Eduardo; Fernando-Navas, Diego; Aponte-Mayor, Guillermo; BetancourtBuitrago, Luis Andrés, (2014). Metodología para la revisión bibliográfica y la gestión de información de temas científicos, a través de su estructuración y sistematización, Dyna, vol. 81, núm. 184, abril, 2014, pp. 158-163, Universidad Nacional de Colombia

[8] Instituto de la tecnología y formación, (2013). Energía solar fotovoltaica, 3ra Edición. Madrid, España.

[9] International Energy Agency, (2016). Solar Heat Worldwide Markets and Contribution to Global Energy Supply. Austria.

[10] ITESM, Instituto Tecnológico de Estudios Superiores de Monterrey, (2015). Gestión y uso eficiente de la energía eléctrica. Monterrey, Nuevo León, México.

[11] Martínez, J, (2012). Dimensionado de instalaciones solares fotovoltaicas. Madrid, España: Paraninfo.

[12] Max Wei, S, (2009). Putting renewable and energy efficiency to work: How many jobs can the clean energy industry generate. California, Estados Unidos: Elsevier.

[13] NASA, Earth Science Enterprise Program, (2015). Surface meteorology and solar energy. Disponible en: https://eosweb.larc.nasa.gov/sse/RETScreen/

[14] Organización de las Naciones Unidas, (2015). Agenda 2030 para el Desarrollo Sostenible, Disponible en: https://academicimpact.un.org/es/content/objetivos-de-desarrollo-sostenible

[15] PNUD, Programa de las Naciones Unidas para el Desarrollo, (2026). ¿Qué son los objetivos de desarrollo sostenible?, Disponible en: https://www.ec.undp.org/content/ecuador/es/home/sustainable-development-goals.html

[16] Romero, J, (2015). Tesina de Máster en Edificación. Análisis del funcionamiento de paneles fotovoltaicos y su utilización. Barcelona, España. 
[17] Salgado, F, (2017). Guía de la energía solar fotovoltaica. Madrid, España.

[18] SENER, (2014). Prospectiva del sector eléctrico americano. Dirección General de Planeación Energética. México, D.F. 230.

[19] Sunfields Europe, (2014). Boletín solar fotovoltaico. Santiago de Compostela, La Coruña, España.

[20] Tous, M, (2010). Energía solar fotovoltaica. Barcelona: Editoriales CEAC.

[21] Vasilis, M, (2017). Photovoltaics systems: Life cycle environmental profile and comparisons, ScienceDirect.

[22] XE, (2020). Convertido oficial de dinero, Disponible en: https://www.xe.com/es/currencyconverter/convert/?Amount=5724\&From=MXN\&To=USD 